\title{
Molecular characterization of multi-drug resistant coagulase negative cocci in non-hospital environment
}

\author{
Daniel Don Nwibo ${ }^{1,2,}$, Suresh Panthee ${ }^{1, \S}$, Hiroshi Hamamoto ${ }^{1}$, Kazuhisa Sekimizu ${ }^{1,3, *}$ \\ ${ }^{1}$ Teikyo University Institute of Medical Mycology, Tokyo, Japan; \\ ${ }^{2}$ Laboratory of Immunology and Microbiology, Graduate School of Pharmaceutical Sciences, The University of Tokyo, Tokyo, \\ Japan; \\ ${ }^{3}$ Genome Pharmaceuticals Institute, Tokyo, Japan.
}

\begin{abstract}
Summary Antibiotic resistance crisis occasioned by sporadic appearance of multi-drug resistance (MDR) in human pathogens to clinically applied antimicrobials is a serious threat to global health. In this study, we investigated the drug resistant phenotype of Gram-positive cocci isolates from environment. Staphylococcus capitis and Staphylococcus haemolyticus colonies were isolated on mannitol-salt agar plates supplemented with tetracycline. Antibiotic susceptibility profile of the isolates via minimum inhibitory concentration (MIC) determination was examined. Isolates showed decreased sensitivity to clinically applied antimicrobial agents: tetracycline, kanamycin, erythromycin, norfloxacin, teicoplanin, and ampicillin. Genomic analysis demonstrated the presence of multiple antibiotic resistant genes in these bacteria, suggesting the origin of the multiple antimicrobials resistant phenotype. Tetracycline resistance of these isolates was transduced to Staphylococcus aureus-RN4220 strain. These findings indicate the presence of multiple antimicrobials resistant $S$. capitis and $S$. haemolyticus strain in a nonhospital setting. Moreover, the presence of plethora of genes responsible for MDR suggest that these strains could present potential threat to human health by serving as reservoir for lateral transference of antimicrobial resistance conferring foreign genetic elements to other clinically relevant pathogens.
\end{abstract}

Keywords: MDR, foreign genetic elements, micro-broth dilution, infectious diseases, antimicrobial resistance determinants, antibiotic resistance crisis

\section{Introduction}

Rampant increase in drug resistance among human pathogens has hampered antimicrobial effectiveness and limited available therapeutic options. Estimated not less than 700,000 people die annually because of antimicrobial resistant infections and is expected to rise to 10 million annual deaths by 2050 costing global economy USD100 trillion if the current trajectory is not halted (1). Understanding the various processes associated with drug resistance may provide important insights into new preventative and therapeutic strategies

\footnotetext{
Released online in J-STAGE as advance publication June 23, 2019.

${ }^{\S}$ These authors contributed equally to this work.

*Address correspondence to:

Dr. Kazuhisa Sekimizu, Teikyo University Institute of Medical Mycology, 359 Otsuka, Hachioji, Tokyo 192-0395, Japan.

E-mail: sekimizu@main.teikyo-u.ac.jp
}

against resistant infections. Antimicrobial resistance (AMR) determinants are present as transposons, integrons or plasmids; which are utility vehicles for genetic transference to other clinically relevant microbes. AMR is complicated by the horizontal gene transfer mechanisms vis-a-vis conjugation, transduction and transformation into clinically relevant pathogens (24 ). Considering the foregoing, accurate identification and proper antimicrobial profiling of pathogenic species are necessary for tracking the epidemiology and treatment of infected patients in health-care settings. The environment is a reservoir of AMR genes and mobile genetic elements that are actively involved in resistant gene mobilization and transfer (5). Environmentallyborne dissidents are key threats to human health because of the increasing relevance of zoonotic diseases and their importance in predicting outbreak of infectious diseases (6-9). Despite all efforts to understand and abrogate AMR, it is evident that all is not well and further 
efforts including scientific investigations are therefore warranted. Coagulase-negative staphylococci (CoNS) are gradually becoming important pathogens associated with nosocomial infections. Staphylococcus capitis, a sub-group of CoNS and a colonizer of human intestinal tract, has been increasingly implicated in several infective diseases including endocarditis, prosthetic valve endocarditis, and late-onset sepsis in very-low birth-weight neonates. $S$. capitis has biofilm production capacity especially on medical placement devices, which contributes to its capacity to invade surgical disinfectants (10). S. haemolyticus is another pathogenic CoNS. Genomic plasticity accounting for several insertion sequences and plasmids some of which confer multiple antibiotics resistance, notably against beta-lactams and glycopeptides in these strains, has been suggested $(11,12)$. As a nosocomial pathogen, S. haemolyticus is primarily a blood culture isolate with capacity to invade immune defenses of compromised individuals. However, data on CoNS isolation from non-hospital environment and genetic resistance mechanisms are sparsely available. In this study, we characterized the molecular mechanisms of multiple antimicrobial resistant $S$. capitis and $S$. haemolyticus strains isolated from nature and experimentally challenged the possibility of horizontal gene transmission to $S$. aureus.

\section{Materials and Methods}

\subsection{Isolation of strains and determination of minimum inhibitory concentration}

Colonies were isolated from the environment using mannitol-salt agar medium plates supplemented with tetracycline; $2 \mu \mathrm{g} / \mathrm{mL}$. Minimum inhibitory concentration (MIC) was determined according to the following procedure. Isolated single colony was cultured overnight in tryptic soy broth medium overnight. Full growth was diluted with sterilized physiological saline to give optical density at $600 \mathrm{~nm}$ (Shimadzu UV-1280 spectrophotometer), 0.5 and re-diluted (1/150). Rediluted culture $(100 \mu \mathrm{L})$ was served with the antibiotics (100 $\mu \mathrm{L})$ into 96 -well plate, two-fold decimally diluted and incubated at $37^{\circ} \mathrm{C}$. Cation-adjusted Mueller Hinton broth (CA-MHB) supplemented with calcium $(25 \mathrm{mg} /$ $\mathrm{mL})$ and magnesium $(12.5 \mathrm{mg} / \mathrm{mL})$ ions were employed for all dilutions. MIC was defined as the lowest concentration of antimicrobial agent that inhibits growth of the organism in the micro-dilution wells as detected by the unaided eye, using the 96-well micro-titer plate format.

\subsection{Genomic DNA isolation, whole genome sequencing and assembly}

Total genomic DNA from $5 \mathrm{~mL}$ full growth was isolated using DNeasy Blood and Tissue kit (Qiagen, Hilden,
Germany) following manufacturer's recommendation. The integrity and purity of the DNA was confirmed by agarose gel electrophoresis and NanoDrop 2000c spectrophotometer (ThermoFisher Scientific, Waltham, MA, USA). Whole genome sequencing was performed using $100 \mathrm{ng}$ of the total DNA as quantified by Qubit 3.0 fluorometer (ThermoFisher) as described previously (13). Briefly, the barcoded library of 400 base-reads was prepared using the Ion Xpress ${ }^{\mathrm{TM}}$ Plus Fragment Library Kit (ThermoFisher). The quality, quantity, and size distribution of the libraries were determined using an Agilent Bioanalyzer 2100 (Agilent Technologies, Santa Clara, CA, United States). The libraries were then enriched in an Ion $318^{\mathrm{TM}}$ Chip v2 using Ion Chef (ThermoFisher), and subsequent sequencing was performed in the Ion PGM System (ThermoFisher). The reads were then assembled using SPAdes 3.11 (14) and further analysis of the assembly was performed using CLC Genomics Workbench ver 11.0 (Qiagen Bioinformatics, Aarhus, Denmark).

\subsection{Conjugation assay}

S. aureus (RN4220 strain) selected with fusidic acid $(6.3 \mu \mathrm{g} / \mathrm{mL})$ and rifampicin $(1 \mu \mathrm{g} / \mathrm{mL})$ was employed as recipients. One $\mathrm{mL}$ full growth (about $10^{9} \mathrm{CFU}$ each) of donors ( $S$. capitis, $S$. haemolyticus) and recipients were mixed on ice, and centrifuged $\left(5,000 \mathrm{rpm}, 4^{\circ} \mathrm{C}, 5 \mathrm{~min}\right)$ and dried on $0.22-\mu \mathrm{m}$ membrane filter (Millipore Sigma, MA-USA). Donors and recipient control experiments were also included. Conjugation was performed on Brain Heart Infusion-agar $(1.5 \%)$ overnight at $37^{\circ} \mathrm{C}$. Cells were recovered in sterilized saline and transconjugants were selected on BHI-agar supplemented with fusidic acid (6.3 $\mu \mathrm{g} / \mathrm{mL})$, rifampicin $(1 \mu \mathrm{g} / \mathrm{mL})$ and tetracycline $(1 \mu \mathrm{g} /$ $\mathrm{mL})$. Incubation at $37^{\circ} \mathrm{C}$ was performed for $48 \mathrm{hs}$.

\subsection{Statistical analysis}

Appearance ratio of transconjugants was compared using Graph Pad Prism 5. ${ }^{*} p<0.01$ was considered statistically significant by unpaired student's $t$-test with $99 \%$ confidence interval.

\subsection{Data availability}

This whole-genome project including the assembled contigs and raw reads has been deposited at NCBI Bio Project under the accession PRJNA471195. The DDBJ/ENA/GenBank accession numbers of each assemblies are: RIYT00000000, RIYU00000000, and RIYV00000000 for $\mathrm{Tc}^{\mathrm{R}}-3, \mathrm{Tc}^{\mathrm{R}}-5$, and $\mathrm{Tc}^{\mathrm{R}}-7$, respectively.

\section{Results}

\subsection{Antibiotic susceptibility profiling of isolates}


Table 1. Identification of isolates

\begin{tabular}{lll}
\hline Strain & Closest homolog & \% identity, gap \\
\hline $\mathrm{Tc}^{\mathrm{R}}-3$ & Staphylococcus capitis strain AYP1020 & $1554 / 1554(100 \%), 0$ \\
$\mathrm{Tc}^{\mathrm{R}}-5$ & Staphylococcus haemolyticus JCSC1435 & $1554 / 1554(100 \%), 0$ \\
$\mathrm{Tc}^{\mathrm{R}}-7$ & Staphylococcus haemolyticus JCSC1435 & $1554 / 1554(100 \%), 0$ \\
\hline To characterize the isolates, & 16S rDNA of whole genome analyzed isolates were extracted and blasted in National Center for Biotechnological
\end{tabular}
Information (NCBI) database.

We focused on the isolation and molecular characterization of environmentally mobilized staphylococci bacteria with antibiotic resistant phenotype. Using tetracycline supplemented mannitol-salt agar medium, we isolated antibiotic resistant bacteria colonies. Cells having mannitol utilization capacity were isolated. Single colony isolation was performed using Brain Heart Infusion-agar plate. To identify the bacteria, we extracted the $16 \mathrm{~S}$ rDNA of the sequenced isolates from the whole genome data. BLAST algorithm was performed in NCBI database. Result suggested that analyzed strains were $S$. capitis and $S$. haemolyticus, with $100 \%$ similarity to available literature data (Table 1). Antimicrobial susceptibility assay of these isolates via minimum inhibitory concentration (MIC) determination was determined. Antibiogramic data (Table 2) showed that the isolates displayed tetracycline resistance. Further examination suggested that the environmental bacteria also displayed decreased sensitivity (elevated MICs) against mechanistically diverse antimicrobial agents compared to CLSI standard breakpoints (15) including norfloxacin, kanamycin, and erythromycin with no observed decreased susceptibility against rifampicin, chloramphenicol and vancomycin. These findings indicate that the isolates possess genetic mechanisms that allow for its existence even in the face of environmental stress.

\subsection{Diversity of antimicrobial resistant genes in the isolates}

To provide genetic evidence and elucidate the molecular mechanisms behind the multidrug resistance of the isolates, we intended to identify the resistant genes in the isolates by whole genome sequencing. Genomic DNA of representative multi-drug resistant isolates $\left(\mathrm{Tc}^{\mathrm{R}}-3, \mathrm{Tc}^{\mathrm{R}}-5\right.$ and $\left.T \mathrm{C}^{\mathrm{R}}-7\right)$ were extracted, sequenced and assembled. Sequence data assembly revealed a genome size range of $2.5 \mathrm{M}$ bp. $\mathrm{G}+\mathrm{C}$ content was approximately $33 \%$. Reads $\left(1.48 \times 10^{6}, 6.03\right.$ $\times 10^{5}$ and $\left.6.44 \times 10^{5}\right)$ and genome coverages $(160,66$, and 73 ) for $T c^{R}-3, T c^{R}-5$, and $T c^{R}-7$ respectively were obtained (Table 3 ). Genome annotation revealed the presence of antibiotic resistance genes: $\operatorname{aac}\left(6^{\prime}\right)-\operatorname{aph}\left(2^{\prime}\right)$, blaZ, $m p h(\mathrm{C}), \operatorname{msr}(\mathrm{A}), \operatorname{vga}(\mathrm{A})$ and $\operatorname{Tet}(\mathrm{K})$ (Table 4).

\subsection{Roles of coagulate negative Staphylococci in spread of antibiotic resistance}

Table 2. Antibiogram of isolated environmental bacteria

\begin{tabular}{lccc}
\hline \multirow{3}{*}{ Antibiotic } & \multicolumn{3}{c}{$\mathrm{MIC}(\mu \mathrm{g} / \mathrm{mL})$} \\
\cline { 2 - 4 } & $\mathrm{Tc}^{\mathrm{R}}-3$ & $\mathrm{Tc}^{\mathrm{R}}-5$ & $\mathrm{Tc}^{\mathrm{R}}-7$ \\
\hline Tetracycline & 128 & 32 & 32 \\
Kanamycin & 128 & 1 & 1 \\
Norfloxacin & 1 & 128 & 128 \\
Erythromycin & 0.5 & 32 & 32 \\
Teicoplanin & 0.5 & 4 & 4 \\
Ampicillin & 1 & 8 & 8 \\
Cefcapene & 1 & 1 & 1 \\
Cefditoren & 0.5 & 0.5 & 0.5 \\
Oxacillin & 0.25 & 0.25 & 0.25 \\
Chloramphenicol & 8 & 4 & 4 \\
Rifampicin & $<0.008$ & $<0.008$ & $<0.008$ \\
Vancomycin & 2 & 1 & 1 \\
\hline
\end{tabular}

Table 3. General feature(s) and genome statistics of the assembled sequences

\begin{tabular}{lrrr}
\hline \multirow{2}{*}{ Feature of chromosome } & \multicolumn{3}{c}{ Value for strain } \\
\cline { 2 - 4 } & $\mathrm{Tc}^{\mathrm{R}}-3$ & $\mathrm{Tc}^{\mathrm{R}}-5$ & $\mathrm{Tc}^{\mathrm{R}}-7$ \\
\hline Length of sequence (bp) & $2,515,208$ & $2,563,958$ & $2,542,447$ \\
G+C content (bp) & 824,989 & 835,851 & 828,838 \\
G+C contents (\%) & 32.8 & 32.6 & 32.6 \\
Number of reads & $1,476,795$ & 603,500 & 643,974 \\
Coverage & 160 & 66 & 73 \\
N50 (bp) & 466,109 & 94,107 & 79,590 \\
L50 (No. of contigs) & 2 & 12 & 9 \\
\hline
\end{tabular}

To understand the role of these isolates in spreading of antibiotic resistance, conjugation assay was performed using $S$. aureus RN4220, a laboratory strain, harboring fusidic acid and rifampicin resistances as recipients. Assay was performed as described in methodology section. Results revealed that the colony-forming units (CFUs) of S. aureus RN4220 cells that appeared on the selection plate after conjugation increased and significantly differed from each recipient controls (Figure 1). Donor controls yielded no colonies on selection plate.

\section{Discussion}

Notwithstanding calls for better picture on the reservoir of genes in our surrounding and how they aid acquisition of resistance phenotypes in clinically relevant pathogens; available genetic information are sparse on the resistome of environmental bacteria. In that regard, this study 
Table 4. Drug resistant genes identified in the assembled genomes

\begin{tabular}{|c|c|c|c|}
\hline Strain & Closest bacteria homolog & Identified gene & Gene function \\
\hline $\mathrm{Tc}^{\mathrm{R}}-3$ & S. capitis & $\begin{array}{l}\operatorname{aac}\left(6^{\prime}\right)-\operatorname{aph}\left(2^{\prime}\right) \\
\operatorname{Tet}(K)\end{array}$ & $\begin{array}{l}\text { Aminoglycoside resistance } \\
\text { Tetracycline resistance }\end{array}$ \\
\hline $\mathrm{Tc}^{\mathrm{R}}-5$ & S. haemolyticus & $\begin{array}{l}\operatorname{blaZ} \\
m p h(C) \\
m s r(A) \\
\operatorname{vga}(A) \\
\operatorname{tet}(K)\end{array}$ & $\begin{array}{l}\text { Beta-lactam resistance } \\
\text { Macrolide resistance } \\
\text { Macrolide, Lincosamide and Streptogramin B resistance } \\
\text { Streptogramin B resistance } \\
\text { Tetracycline resistance }\end{array}$ \\
\hline $\mathrm{Tc}^{\mathrm{R}}-7$ & S. haemolyticus & $\begin{array}{l}\operatorname{blaZ} \\
\operatorname{mph}(C) \\
\operatorname{msr}(A) \\
\operatorname{vga}(A) \\
\operatorname{tet}(K)\end{array}$ & $\begin{array}{l}\text { Beta-lactam resistance } \\
\text { Macrolide resistance } \\
\text { Macrolide, Lincosamide and Streptogramin B resistance } \\
\text { Streptogramin B resistance } \\
\text { Tetracycline resistance }\end{array}$ \\
\hline
\end{tabular}

The nucleotide sequences of the assembled genomes were analyzed in the CLC Genomics Workbench against the database of Find Resistance using default parameters.

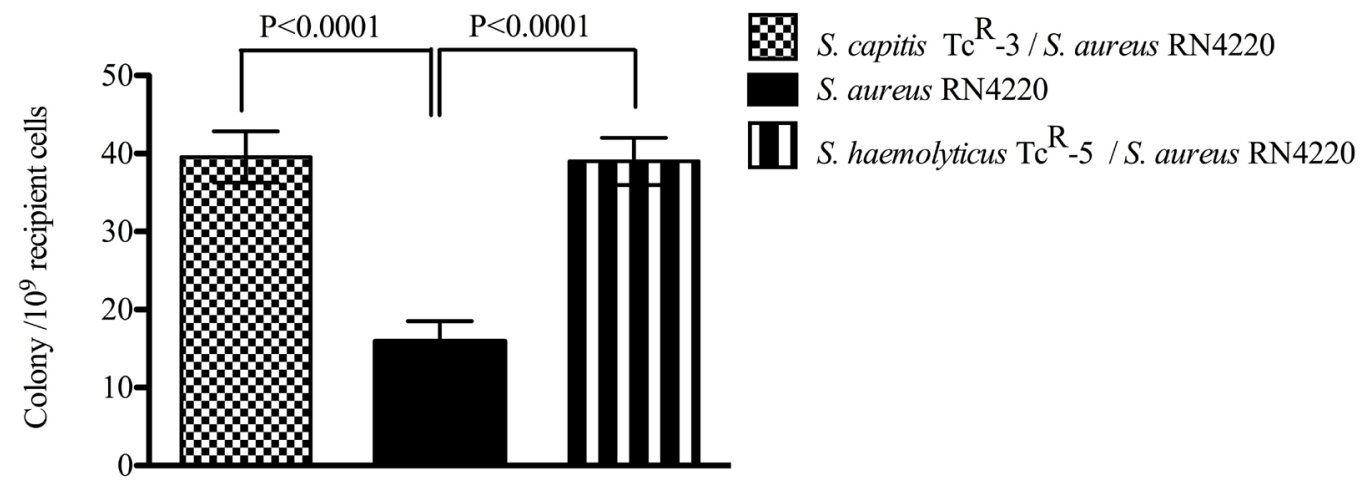

Figure 1. Conjugational transduction of tetracycline resistance to $S$. aureus. Conjugation assay was performed on BHIagar media. To determine total number of recipient present after conjugation, recovered suspensions were diluted and spread on selective media plate and ratio of transconjugants to total recipient cells determined after $48 \mathrm{hrs}$. Experiments were repeated thrice in triplicates. Plots show mean and standard deviation. Statically analysis was performed by unpaired Student's $t$-test with $99 \%$ confidence interval.

investigated multiple antimicrobials resistant bacteria isolated from a non-clinical environment in Japan. In addition to clinically relevant antimicrobial agents, these strains can transport environmental biocides and disinfectants which may allow persistence in the environment and subsequent transmission on to a suitable host. Molecular analysis of three isolates from the library showed the presence of multiple antibiotic resistant genes: $\operatorname{aac}\left(6^{\prime}\right)-\operatorname{aph}\left(2^{\prime}\right)$, blaZ, $m p h(\mathrm{C})$, $m s r(\mathrm{~A})$, and $\operatorname{Tet}(\mathrm{K})$ suggetsing the origin of the MDR phenotype. Decreased sensitivity to tetracycline in staphylococci mediated by expression of a ribosomal protection protein molecule encoded by $\operatorname{Tet}(\mathrm{K})$ or Tet(M) genes, or via efflux mechanisms has been investigated (17). Aminoglycoside-modifying enzyme gene, $a a c\left(6^{\prime}\right)-a p h$, is the most common antimicrobial resistant gene conferring aminoglycoside, including kanamycin resistance, in staphylococci. The capacities of the blaZ and $\operatorname{msr}(\mathrm{A})$ genes in conferring betalactams and macrolide resistances respectively are well-known. In particular, $\operatorname{msr}(\mathrm{A})$ gene encode ATPdependent efflux pump conferring resistance to 14 - and 15-membered macrolides, including erythromycin. Still, none of the identified genes is associated with decreased sensitivity to norfloxacin and teicoplanin. The $m p h(\mathrm{C})$ and $m s r(\mathrm{~A})$ are key candidates genes, though none is previously associated with efflux of fluoroquinolones or glycopeptides. Further studies are required to elucidate the actual resistance mechanisms. This data supports the need for the controlled use of antibiotics and establishes the criticality of CoNS as a reservoir of antibiotic resistant determinants. In the knowledge of the authors, this is the first study to document the isolation of $S$. capitis harboring multiple antibiotic resistant genes from non-clinical (nonhospital) environment. Despite untiring efforts towards analyses of resistant clinical bacterial isolates, the exact process of the acquisition of resistance to these antimicrobials remains unclear. For environmental microbial resistant armamentarium, the stories are not different and further studies are thus necessitated. Understanding these mechanisms could potentially aid antimicrobial agents development. Presence of plethora of genes responsible for antibiotic resistance suggests 
that strains $S$. haemolyticus and $S$. capitis could present a potential threat to human health. Environmentally mobilized MDR bacteria such S. haemolyticus and $S$. capitis might serve as a foothold towards transference of AMR conferring foreign genetic elements into other clinically relevant pathogens.

\section{Acknowledgements}

This work was supported in part by a Grant-in-Aid for scientific research (S) (JP15H05783) to KS; a Grant-inAid for JSPS Fellows (JP17F17421) to SP and KS; and Japanese Government Scholarship through the Ministry of Education, Culture, Sports and Technology (MEXT) to NDD. We thank Dr. Yasuhiko Matsumoto (Institute of Medical Mycology Teikyo University, Tokyo), Dr. Masaki Ishii (Graduate School of Pharmaceutical Sciences, Musashino University, Tokyo) for fruitful discussions and staffs of Genome Pharmaceuticals Institute for technical support.

\section{Conflict of Interest}

K.S. is a consultant for Genome Pharmaceuticals Institute Co., Ltd.

\section{References}

1. O'Neill J. Tackling drug-resistant infections globally: Final Report and Recommendations. The review on antimicrobial resistance. UK Government sponsored, 2016; https://amr-review.org/sites/default/files/160518_ Final\%20paper_with\%20cover.pdf (accessed June 26, 2018).

2. Crofts TS, Gasparrini AJ, Gautam Dantas G. Nextgeneration approaches to understand and combat the antibiotic resistome. Nat Rev Microbiol. 2017; 15:422434.

3. Thomas CM, Nielsen KM. Mechanisms of, and barriers to, horizontal gene transfer between bacteria. Nat Rev Microbiol. 2005; 711-721.

4. Wolska KI. Horizontal DNA transfer between bacteria in the environment. Acta Microbiol Pol. 2003; 52:233-243.

5. Singer AC, Shaw H, Rhodes V, Hart A. Review of antimicrobial resistance in the environment and its relevance to environmental regulators. Front Microbiol. 2016; 7:1728.

6. Huijbers PM, Blaak H, de Jong MC, Graat EA, Vandenbroucke-Grouls CM, de Roda Husman AM. Role of the environment in the transmission of antimicrobial resistance to humans: A review. Environ Sci Technol. 2015; 49:11993-12004.

7. Cantas L, Shah SQ, Cavaco LM, Manaia CM, Walsh F, Popowska M, Garelick H, Bürgmann H, Sørum H. A brief multi-disciplinary review on antimicrobial resistance in medicine and its linkage to the global environmental microbiota. Front Microbiol. 2013; 4:96.

8. Allen HK, Donato J, Wang HH, Cloud-Hansen KA, Davies J, Handelsman J. Call of the wild: Antibiotic resistance genes in natural environments. Nat Rev Microbiol. 2010; 8:251-259.

9. Larsson DGJ, Andremont A, Bengtsson-Palme J, et al. Critical Knowledge gaps and research needs related to enviornmental dimensions of antibiotic resistance. Environ Int. 2018; 117:132-138.

10. Li X, Lei M, Song Y, Gong K, Li L, Liang H, Jiang $\mathrm{X}$. Whole genome sequence and comparative genomic analysis of multidrug-resistant Staphylococcus capitis subsp. urealyticus strain LNZR-1. Gut Pathog. 2014; 6:45.

11. Takeuchi F, Watanabe S, Baba T, Yuzawa H, Teruyo Ito T, Morimoto Y, Kuroda M, Cui L, Takahashi M, Ankai A, Baba S, Fukui S, Lee JC, Hiramatsu K. Whole-genome sequencing of Staphylococcus haemolyticus uncovers the extreme plasticity of its genome and the evolution of human-colonizing staphylococcal species. J Bacteriol. 2005; 187:7292-7308.

12. Froggatt JW, Johnston JL, Galetto DW, Archer GL. Antimicrobial resistance in nosocomial isolates of Staphylococcus haemolyticus. Antimicrob Agents Chemother. 1989; 33:460-466.

13. Panthee S, Hamamoto H, Ishijima SA, Paudel A, Sekimizu K. Utilization of hybrid assembly approach to determine the genome of an opportunistic pathogenic fungus, Candida albicans TIMM 1768. Genome Biol Evol. 2018; 10:2017-2022.

14. Bankevich A, Nurk S, Antipov D, et al. SPAdes: A new genome assembly algorithm and its applications to singlecell sequencing. J Comp Biol. 2012; 19:455-477.

15. Clinical and Laboratory Standards Institute. Performance Standards for Antimicrobial Susceptibility Testing; Twenty-Fifth Informational Supplement. CLSI document M100-S25. Wayne, PA, USA, 2015.

16. Akimitsu N, Hamamoto H, Inoue R, Shoji M, Akamine A, Takemori K, Hamasaki N, Sekimizu K. Increase in resistance of MRSA to $\beta$-lactams caused by mutations conferring resistance to BKC, a disinfectant widely used in hospitals. Antimicrob Agents Chemother. 1999; 43:3042-3044

17. Ardic N, Ozyurt M, Sareyyupoglu B, Haznedaroglu T. Investigation of erythromycin and tetracycline resistance genes in methicillin-resistant staphylococci. Int J Antimicrob Agents. 2005; 26:213-215.

(Received May 15, 2019; Accepted June 7, 2019) 